\title{
Study on the Influence of Aerosol Radiation Balance in One-Dimensional Atmospheric Medium Using $P_{n}$-Approximation Method
}

\author{
Yuan Yuan, ${ }^{1}$ Xing Huang, ${ }^{1}$ Yong Shuai, ${ }^{1}$ and Qian-Jun Mao ${ }^{2}$ \\ ${ }^{1}$ School of Energy Science and Engineering, Harbin Institute of Technology, Harbin 150001, China \\ ${ }^{2}$ School of Civil Engineering and Architecture, Northeast Petroleum University, Daqing 163318, China \\ Correspondence should be addressed to Yuan Yuan; yuanyuan83@hit.edu.cn and Yong Shuai; shuaiyong@hit.edu.cn
}

Received 17 May 2014; Revised 10 July 2014; Accepted 20 July 2014; Published 27 August 2014

Academic Editor: Hong-Liang Yi

Copyright (C) 2014 Yuan Yuan et al. This is an open access article distributed under the Creative Commons Attribution License, which permits unrestricted use, distribution, and reproduction in any medium, provided the original work is properly cited.

A numerical study, used for the influence of aerosol on the atmospheric radiation transfer, is conducted in this paper. Based on the established atmospheric radiation transfer model, we calculated the effect of species, concentration, and height distribution of aerosols on atmosphere radiation. The calculation results show that the aerosol particles affect the atmospheric radiation balance greatly and thus are the important component of the radiation balance of the earth-troposphere system.

\section{Introduction}

Atmospheric aerosols are suspensions of small solid or liquid particles with the diameters between 0.001 and $10 \mu \mathrm{m}$. Aerosol affects the Earth's radiation budget by direct and indirect effects and plays an important role in global climate systems. The direct effect is that aerosol particles scatter and absorb the solar radiation and surface infrared radiation [1]; the indirect effect is that aerosol particles act as cloud condensation nuclei and ice nuclei $[2,3]$. When the climate system is in balance, its absorbing solar radiation energy is accurately equal to the infrared radiation energy that the earth and atmosphere emit into outer space. Any factor that disturbs this balance and then changes the climate is the radiative forcing factor. Its forcing on the earth-troposphere system is the radiative forcing $[4,5]$. Radiative forcing (in $\mathrm{W} \cdot \mathrm{m}^{-2}$ ) is defined as the change in the net radiative flux at the tropopause due to some factors change. Aerosol is a very important correction factor in the atmospheric radiation transfer. So it is significant to study the influence of aerosols particles on the atmospheric transfer.
Many numerical methods have been developed to solve the problem of radiative heat transfer, such as the discrete ordinates method (DOM) [6, 7], finite volume method (FVM) [8], finite element method (FEM), Monte Carlo method (MCM) [9], and spherical harmonics method (SHM). The spherical harmonics method, also known as the $P_{n}$-approximation method, was originally proposed by Jeans [10] for interstellar radiative transfer and further developed by Davison [11] and Kourganoff [12] for neutron transport. Khouaja et al. [13] developed the $P_{n}$-approximation method for radiative heat transfer. Recently, Larsen et al. [14] used a double- $P_{n}$ approximation for the transfer equation in neutron transport, but only to low order in the expansion. McClarren et al. [15] analyzed numerical oscillations of the $P_{n}$-approximation method and also suggested improvements based on wavelet analysis.

We established atmospheric radiative transfer calculations model based on the spherical harmonics method in onedimensional media and analyzed the influence of aerosols on the atmospheric radiative transfer. The calculations from the species, number, and distribution height of aerosols were achieved and the results were given in Figure 1. 


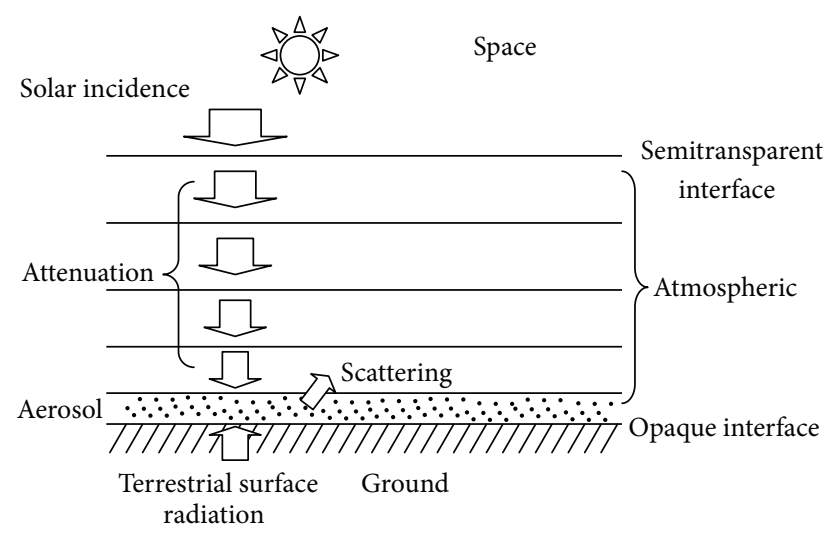

FIGURE 1: Radiative heat transfer model diagram of one-dimensional atmospheric layer.

\section{Numerical Model}

The radiative transfer equation for one-dimensional absorbing, emitting, and scattering gray medium can be expressed as

$$
\begin{aligned}
\mu \frac{d I(\tau, \mu)}{d \tau}= & -I(\tau, \mu)+(1-\omega) I_{b}(\tau) \\
& +\frac{\omega}{2} \int_{-1}^{1} I\left(\tau, \mu^{\prime}\right) \Phi\left(\mu^{\prime}, \mu\right) d \mu^{\prime},
\end{aligned}
$$

where $\tau$ is the optical thickness given by $\tau=\left(\kappa_{a}+\kappa_{s}\right) L$, $\omega$ is the scattering albedo with $\omega=\kappa_{s} /\left(\kappa_{a}+\kappa_{s}\right), L$ is the medium thickness, and $\kappa_{a}$ and $\kappa_{s}$ are the absorbing and scattering coefficients, respectively. The radiative intensity $I(\tau, \mu)$ and scattering phase function $\Phi\left(\mu^{\prime}, \mu\right)$ can be expanded in terms of associated Legendre polynomials $P_{n}(\mu)$. Using recurrence relations between these polynomials, (1) becomes

$$
\begin{gathered}
\frac{d}{d \tau}\left(\sum_{n=0}^{N} \frac{(n+1) P_{n+1}(\mu)+n P_{n-1}(\mu)}{2 n+1} I_{n}(\tau)\right) \\
=-\sum_{n=0}^{N} I_{n}(\tau) P_{n}(\mu)+(1-\omega) I_{b}(\tau) \\
+\sum_{n=0}^{N} \frac{\omega g_{n}}{2 n+1} I_{n}(\tau) P_{n}(\mu),
\end{gathered}
$$

where $g_{n}$ is an asymmetry parameter. Multiplying (2) by $P_{n}(\mu)$ and integrating over $\mu \in[-1,1]$, we obtain using the orthogonality properties of the Legendre polynomials $N+$ 1 differential equations for coefficients $I_{n}(\tau)$ that comprise the $N+1 P_{n}$-approximation equations. Consider the following:

$$
\begin{gathered}
\frac{1}{3} \frac{d I_{1}(\tau)}{d \tau}+\left(1-\omega g_{0}\right) I_{0}(\tau)=(1-\omega) I_{b}(\tau) \quad n=0 \\
\frac{2}{5} \frac{d I_{2}(\tau)}{d \tau}+\frac{d I_{0}(\tau)}{d \tau}+\left(1-\frac{1}{3} \omega g_{1}\right) I_{1}(\tau)=0 \quad n=1 \\
\ldots \\
\frac{n+1}{2 n+3} \frac{d I_{n+1}(\tau)}{d \tau}+\frac{n}{2 n-1} \frac{d I_{n-1}(\tau)}{d \tau}+\left(1-\frac{\omega g_{n}}{2 n+1}\right) I_{n}(\tau) \\
=(1-\omega) I_{b}(\tau) \delta_{0 n} \quad n=2,3, \ldots, N .
\end{gathered}
$$

For one-dimensional opaque diffuse emitting and reflecting interfaces, odd terms in the Legendre polynomials form an orthogonal set. The boundary conditions are given by

$$
\begin{aligned}
& \int_{\overrightarrow{\mathbf{n}} \cdot \overrightarrow{\mathbf{s}}>0} I\left(\tau_{W=0}, \overrightarrow{\mathbf{s}}\right) P_{2 n+1}(\overrightarrow{\mathbf{s}}) d \Omega \\
& =\varepsilon_{W} \int_{\overrightarrow{\mathbf{n}} \cdot \overrightarrow{\mathbf{s}}>0} I_{W}(0, \overrightarrow{\mathbf{s}}) P_{2 n+1}(\overrightarrow{\mathbf{s}}) d \Omega \\
& \quad+\int_{\overrightarrow{\mathbf{n}} \cdot \overrightarrow{\mathbf{s}}>0}\left(1-\varepsilon_{W}\right) \frac{1}{2 \pi} \int_{\overrightarrow{\mathbf{n}} \cdot \overrightarrow{\mathbf{s}}<0} I\left(\tau_{W}, \overrightarrow{\mathbf{s}}^{\prime}\right) d \Omega^{\prime} P_{2 n+1}(\overrightarrow{\mathbf{s}}) d \Omega,
\end{aligned}
$$

where $\varepsilon$ is the emissivity. The boundary conditions imposed on the spherical harmonics functions are given by

$$
\begin{aligned}
& \sum_{n=0}^{N} I_{n}(0)\left[\int_{0}^{1} P_{n}(\mu) \mu^{2 n+1} d \mu\right. \\
& \left.\quad-\rho^{d} \int_{0}^{1} \int_{-1}^{0} P_{n}\left(\mu^{\prime}\right) \mu^{2 n+1} d \mu^{\prime} d \mu\right] \\
& \quad=I_{b 0}\left(1-\rho_{0}^{d}\right) \int_{0}^{1} \mu^{2 n+1} d \mu \\
& \sum_{n=0}^{N} I_{n}\left(\tau_{L}\right)\left[\int_{-1}^{0} P_{n}(\mu) \mu^{2 n+1} d \mu\right. \\
& \left.\quad-\rho^{d} \int_{-1}^{0} \int_{0}^{1} P_{n}\left(\mu^{\prime}\right) \mu^{2 n+1} d \mu^{\prime} d \mu\right] \\
& =I_{b \tau_{L}}\left(1-\rho_{0}^{d}\right) \int_{-1}^{0} \mu^{2 n+1} d \mu \\
& n=0,1, \ldots, \frac{N-1}{2} .
\end{aligned}
$$


The boundary conditions [16] are applicable in the onedimensional case:

$$
\begin{aligned}
& \int_{\overrightarrow{\mathbf{n}} \cdot \overrightarrow{\mathbf{s}}>0} I\left(\tau_{W=0}, \overrightarrow{\mathbf{s}}\right) P_{2 n+1}(\overrightarrow{\mathbf{s}}) d \Omega \\
& =\left(1-\rho_{0}^{d}\right) \int_{\overrightarrow{\mathbf{n}} \cdot \overrightarrow{\mathbf{s}}>0} I^{0}(\overrightarrow{\mathbf{s}}) P_{2 n+1}(\overrightarrow{\mathbf{s}}) d \Omega \\
& \quad+\int_{\overrightarrow{\mathbf{n}} \cdot \overrightarrow{\mathbf{s}}>0} \rho^{d} \frac{1}{2 \pi} \int_{\overrightarrow{\mathbf{n}} \cdot \overrightarrow{\mathbf{s}}<0} I\left(\tau_{W}, \overrightarrow{\mathbf{s}}^{\prime}\right) d \Omega^{\prime} P_{2 n+1}(\overrightarrow{\mathbf{s}}) d \Omega,
\end{aligned}
$$

where $I^{0}(\overrightarrow{\mathbf{s}})$ is the circumstance radiation, $\rho_{0}^{d}$ and $\rho^{d}$ are reflectivities of the two sides of the boundary surfaces, respectively, when considering total reflection, and the values of these depend on the medium refractive index $n$.

Separating the large refractive index from small, the diffuse reflectance at the boundary surfaces is given by Siegel and Spuckler [17]:

$$
\begin{aligned}
\rho(n) \equiv F(n)= & \frac{1}{2}+\frac{(3 n+1)(n-1)}{6(n+1)^{2}} \\
& +\frac{n^{2}\left(n^{2}-1\right)^{2}}{\left(n^{2}+1\right)^{3}} \ln \left(\frac{n-1}{n+1}\right)-\frac{2 n^{3}\left(n^{2}+2 n-1\right)}{\left(n^{2}+1\right)\left(n^{4}-1\right)} \\
& +\frac{8 n^{4}\left(n^{4}+1\right)}{\left(n^{2}+1\right)\left(n^{4}-1\right)^{2}} \ln (n) .
\end{aligned}
$$

In contrast, separating small refractive index from large, the diffuse reflectance at the boundary surfaces is given by

$$
\rho(n)=1-\frac{1}{n^{2}}[1-F(n)],
$$

where $n=\left(n_{h} / n_{s}\right), n_{h} \geq n_{s}$. In treating a semitransparent interface, we can use opaque interface treatment as reference.

Considering one-dimensional unsteady coupled radiative and conductive heat transfer, the general form of the energy equation of the internal radiation and the transient coupled conductive heat transfer is defined as

$$
\rho c \frac{\partial T}{\partial t}=\operatorname{div}\left(k_{c} \operatorname{grad} T\right)-\operatorname{div} \mathbf{q}^{r}+\phi .
$$

Considering unsteady state, no internal heat source and coupled radiative and conductive heat transfer, the energy equation (9) can be discretized as follows:

$$
\begin{aligned}
\rho c \Delta & x_{i} \frac{T_{i}^{m+1}-T_{i}^{m}}{\Delta t} \\
= & \frac{k_{e}\left(T_{i+1}^{m+1}-T_{i}^{m+1}\right)+k_{w}\left(T_{i-1}^{m+1}-T_{i}^{m+1}\right)}{\Delta x_{i}}+\Phi_{i, m}^{r} .
\end{aligned}
$$

In order to make the problem have universal significance, the dimensionless form of the energy equation (10) will be evaluated. Introducing the following definitions: reference temperature $T_{r f}$, the dimensionless temperature $\widetilde{T}_{i}^{m}=$ $T_{i}^{m} / T_{r f}$, unit heat capacity $C=\rho c$, the dimensionless time $\Delta \widetilde{t}=\left(4 \sigma n^{2} T_{r f}^{3} / C L\right) \Delta t, \tilde{t}=\left(4 \sigma n^{2} T_{r f}^{3} / C L\right) t$, the dimensionless length $\Delta X_{i}=\Delta x_{i} / L$, the radiative and conductive parameter $N=\lambda / 4 \sigma n^{2} T_{r f}^{3} L$, the dimensionless convective heat transfer coefficient $H=h / 4 \sigma n^{2} T_{r f}^{3}$, the dimensionless radiative heat flux $\widetilde{\Phi}=\Phi /\left(4 \sigma n^{2} T_{r f}^{4}\right)$, (10) becomes

$$
\begin{aligned}
\Delta X_{i} \frac{\widetilde{T}_{i}^{m+1}-\widetilde{T}_{i}^{m}}{\Delta \widetilde{t}} & \\
& =\frac{N_{e}\left(\widetilde{T}_{i+1}^{m+1}-\widetilde{T}_{i}^{m+1}\right)+N_{w}\left(\widetilde{T}_{i-1}^{m+1}-\widetilde{T}_{i}^{m+1}\right)}{\Delta X_{i}}+\widetilde{\Phi}_{i, m}^{r} .
\end{aligned}
$$

According to [18], in considering one-dimensional nonsteady coupled radiative and conductive heat transfers, the thermal radiation source term $\Phi^{r}(T)$ is found to be

$$
\boldsymbol{\Phi}^{r}(T)=\int_{\Delta V}-\operatorname{div} \mathbf{q}^{r} d V=\int_{\Delta V}-\kappa_{a}\left[4 \pi I_{b}(\tau)-G(\tau)\right] d V .
$$

Here, the incident radiative power $G(\tau)=\int_{\Omega} I(\tau, \Omega) d \Omega$ can be transformed to $G(\tau)=2 \pi \int_{-1}^{1} I(\tau, \mu) d \mu$. According to the properties of Legendre polynomials and $P_{0}(\mu)=1$, the above equation is rearranged into the form:

$$
G(\tau)=2 \pi \int_{-1}^{1} I_{0}(\tau) d \mu .
$$

Meanwhile, considering the physical properties of the inhomogeneous medium, we next develop a $P_{n}{ }^{-}$ approximation method for a multilayer medium. We redetermine the boundary conditions of each layer. The modified boundary conditions are

$$
\begin{gathered}
\sum_{n=0}^{N} I_{n}(0)\left[\int_{0}^{1} P_{n}(\mu) \mu^{2 i+1} d \mu-\rho^{d} \int_{0}^{1} \int_{-1}^{0} P_{n}\left(\mu^{\prime}\right) \mu^{2 i+1} d \mu^{\prime} d \mu\right] \\
=\sum_{n=0}^{N} I_{n, b 0}\left(1-\rho_{0}^{d}\right) \int_{0}^{1} P_{n}(\mu) \mu^{2 i+1} d \mu, \\
\sum_{n=0}^{N} I_{n}\left(\tau_{L}\right)\left[\int_{-1}^{0} P_{n}(\mu) \mu^{2 i+1} d \mu-\rho^{d} \int_{-1}^{0} \int_{0}^{1} P_{n}\left(\mu^{\prime}\right) \mu^{2 i+1} d \mu^{\prime} d \mu\right] \\
=\sum_{n=0}^{N} I_{n, b \tau_{\lambda}}\left(1-\rho_{0}^{d}\right) \int_{-1}^{0} P_{n}(\mu) \mu^{2 i+1} d \mu, \\
i=0,1, \ldots, \frac{N-1}{2} .
\end{gathered}
$$


TABLE 1: The main input parameters of the atmospheric radiation calculation.

\begin{tabular}{|c|c|c|}
\hline & Parameters & Values \\
\hline \multirow{4}{*}{ Initial condition } & Initial temperature $T_{0}$ & $280 \mathrm{~K}$ \\
\hline & Reference temperature $T_{\mathrm{rf}}$ & $1000 \mathrm{~K}$ \\
\hline & Dimensionless time $t^{*}$ & $1 \times 10^{6}$ \\
\hline & Relaxation factor $f_{\text {re }}$ & 0.01 \\
\hline \multirow{4}{*}{ Boundary condition } & Terrestrial temperature $T_{-\infty}$ & $280 \mathrm{~K}$ \\
\hline & Terrestrial emissivity $\varepsilon_{-\infty}$ & 0.7 \\
\hline & Solar radiation temperature $T_{+\infty}$ & $5762 \mathrm{~K}$ \\
\hline & Angle factor $A_{+\infty, 2}$ & $5.5844 \times 10^{-6}$ \\
\hline \multirow{3}{*}{ Media physical properties } & Layers of height & 7 layers, seen in Table 3 \\
\hline & Bands absorption coefficient & 12 bands, seen in Table 4 \\
\hline & Thermal conductivity $k_{c}$ & $0.023 \mathrm{~W} \cdot \mathrm{m}^{-1} \cdot \mathrm{K}^{-1}$ \\
\hline \multirow{2}{*}{ Aerosol properties } & Distribution function & Multilognormal distributions \\
\hline & Height distribution & $0-1 \mathrm{~km}$ \\
\hline
\end{tabular}

TABLE 2: Multilayer atmospheric radiative transfer model.

\begin{tabular}{|c|c|c|c|c|c|}
\hline Layer number & Thickness (km) & $\begin{array}{c}\text { Upper-lower limit of } \\
\text { height }(\mathrm{km})\end{array}$ & Density $\left(\mathrm{kg} / \mathrm{m}^{3}\right)$ & $\begin{array}{c}\text { Dimensionless } \\
\text { thermal conductivity }\end{array}$ & Refractive index \\
\hline 1 & 1 & $0 \sim 1$ & 1.15566 & $7.51 e-8$ & 1.0 \\
\hline 2 & 2 & $1 \sim 3$ & 0.96517 & $5.89 e-8$ & 1.0 \\
\hline 3 & 6 & $3 \sim 9$ & 0.60438 & $3.74 e-8$ & 1.0 \\
\hline 4 & 8 & $9 \sim 17$ & 0.30815 & $1.43 e-8$ & 1.0 \\
\hline 5 & 10 & $17 \sim 27$ & 0.02573 & $3.23 e-9$ & 1.0 \\
\hline 6 & 11 & $27 \sim 38$ & 0.010076 & $6.90 e-10$ & 1.0 \\
\hline 7 & 12 & $38 \sim 50$ & 0.002195 & $4.23 e-10$ & 1.0 \\
\hline
\end{tabular}

Here, $I_{n, b 0}$ and $I_{n, b \tau_{\lambda}}$ are the emission radiation intensities of two adjacent layers, and the initial values are obtained from the temperature field. The total emission radiation intensity is determined by an iterative calculation of intensities from successive layers, the last providing the required total. The convergence condition of the multilayer model calculation is decided by the radiative intensity $I_{n}$. After the intensity calculation has converged, the iterative calculation of the temperature field is then performed.

\section{Results and Discussion}

According to statistics, $98.8 \%$ of the total atmospheric mass distributes in the atmosphere within $30 \mathrm{~km}$ above the ground, and with the height increasing to $50 \mathrm{~km}$ the proportion reaches $99.92 \%$. Most of the atmospheric radiation (involving solar radiation, absorption, and scattering of the ground infrared radiation and the emission of the atmosphere itself) occurs within certain atmospheric thickness. Compared with the earth radius $(6378 \mathrm{~km})$, the atmospheric thickness can be regarded as one-dimensional radiative media. Therefore, we treated the atmosphere within $50 \mathrm{~km}$ above the ground as a multilayer one-dimensional media distributing along the radial of the earth. The upper surface of the medium layer is near to space and is considered as a semitransparent medium. The lower interface is close to ground and is considered as opaque interface. The emissivity and radiative temperature related to the ground are given. For the inner of the medium layer we mainly consider the gas absorption and emission of the radiation energy and the aerosol particles scattering and absorption of the radiation energy and consider the atmospheric coupled radiation and conduction in the vertical direction.

The atmospheric convection is very important for the energy transfer of the atmosphere. But we mainly discuss the influence of aerosols on the atmospheric radiative energy and investigate the atmospheric radiative balance and the variation in radiation characteristic, which is not affected by the atmospheric convection that acts only as the background. Therefore, we ignore the influence of the atmospheric convection.

The atmospheric radiation physical model is shown in Figure 1 and the atmospheric radiation transfer model is shown in Figure 2. The atmospheric internal heat source is set as $\phi=0$.

The standard atmospheric condition is given in Tables 1-4.

Table 1 gives the main input parameters for the calculation of atmospheric radiation transfer.

Table 2 gives the parameters associated with layers divided along the vertical height. The density of each layer is determined by the calculation of the pressure. We consider $\left(\rho / \rho_{0}\right)=\left(p / p_{0}\right)$ (the standard atmosphere pressure $p$ is 


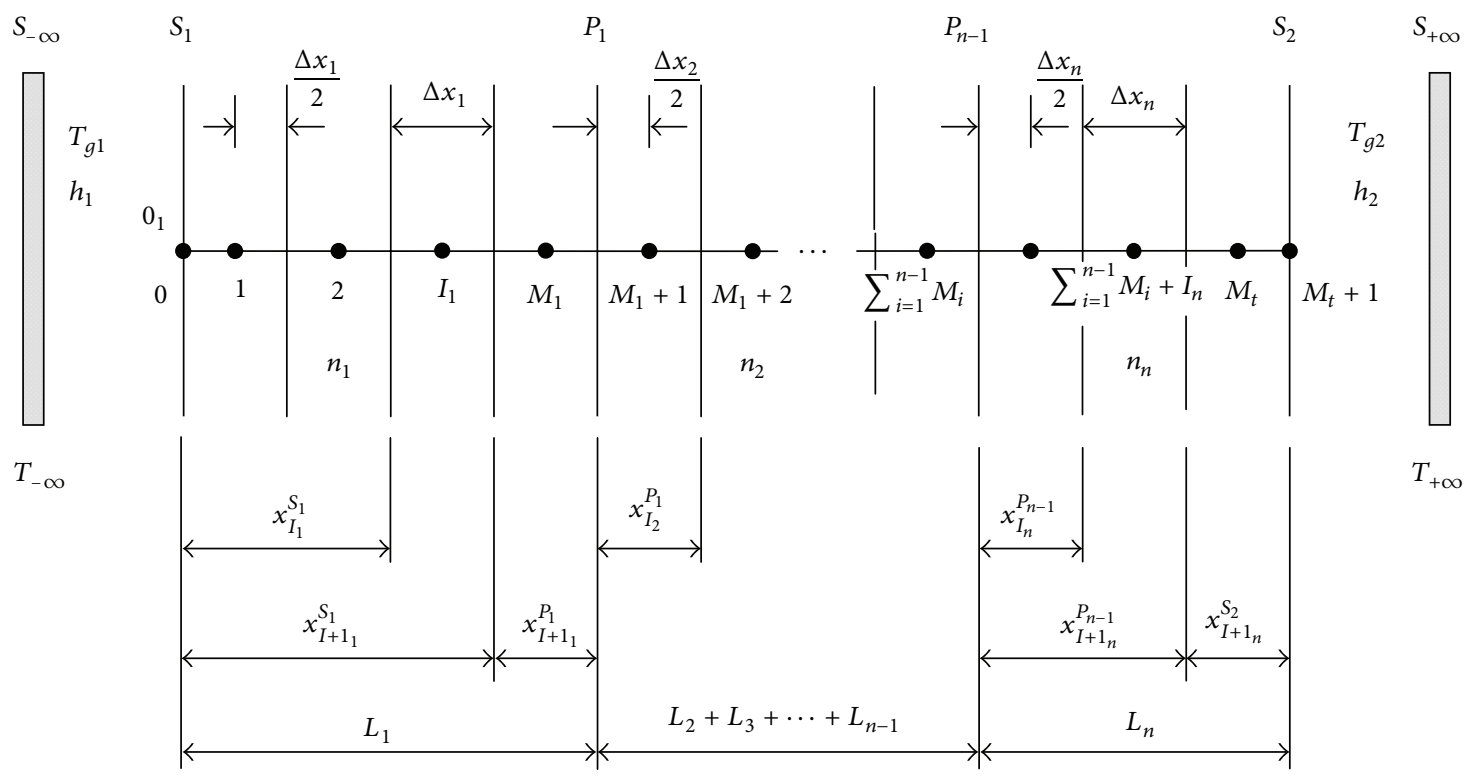

FIgURE 2: Physical model of one plane-parallel slab divided into $n$ sublayers.

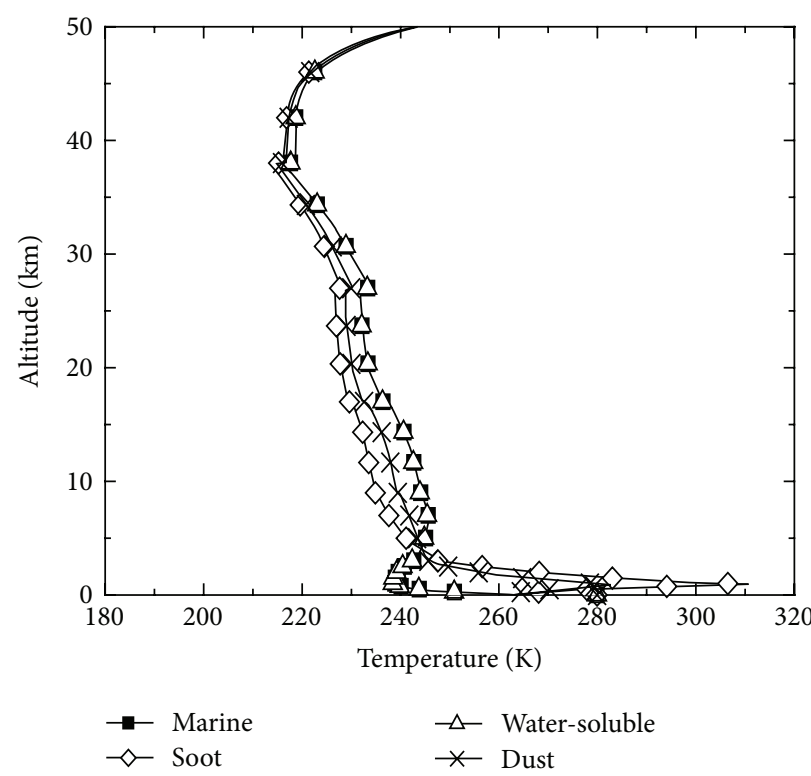

(a) Atmosphere temperature field $(0-50 \mathrm{~km})$

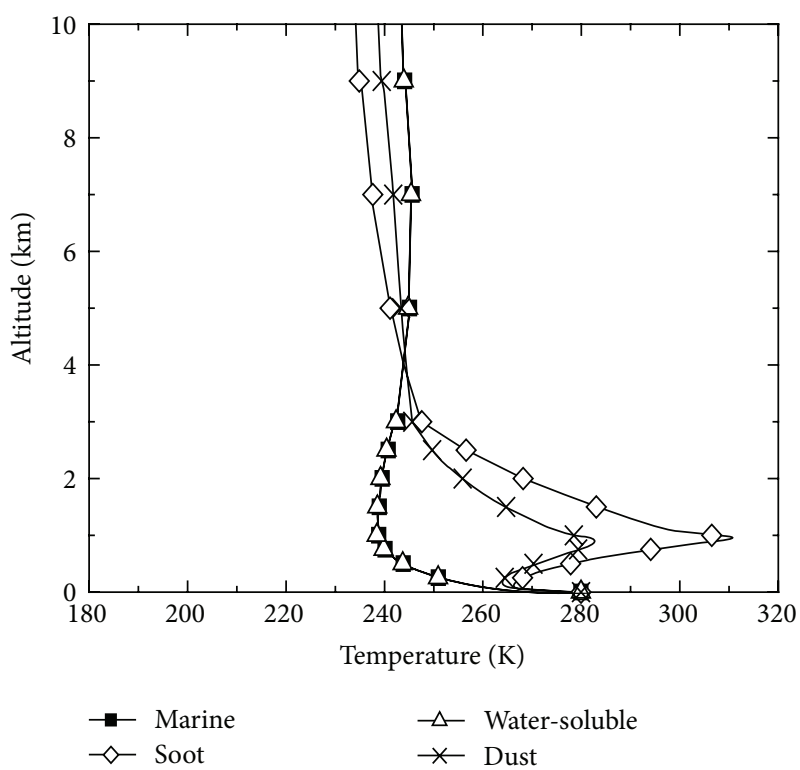

(b) Atmosphere temperature field $(0-10 \mathrm{~km})$

FIGURE 3: Influence of aerosols species on temperature fields.

$1.225 \mathrm{~kg} / \mathrm{m}^{2}$ ) with the temperature fluctuating in small range. Therefore, the equation is as follows:

$$
\rho=\frac{\rho_{0}}{p_{0}} \cdot p=\frac{1.225}{101325} \cdot p=1.20898 \times 10^{-5} \cdot p .
$$

Table 3 gives the wavelength range of each spectral band. The atmosphere optical thicknesses of the layers at different spectral bands are obtained from the HITRAN database under the standard atmospheric condition of America in 1976. The aerosol optical thicknesses are decided based on the Mie scattering theory using the typical aerosol particles size distribution assumption and combining the complex refractive index of different aerosol species. Table 3 lists the spectral optical thicknesses of the dust aerosol and the scattering albedo parameters in the height of $0-1 \mathrm{~km}$. The other parameters are not given in this paper as there are too much data.

Table 4 gives the complex refractive index of different aerosols.

3.1. The Influence of Aerosol Species Variation. Figure 3 shows the influence of four aerosol species (dust, soot, marine, and water-soluble) on the atmospheric temperature. The aerosol 


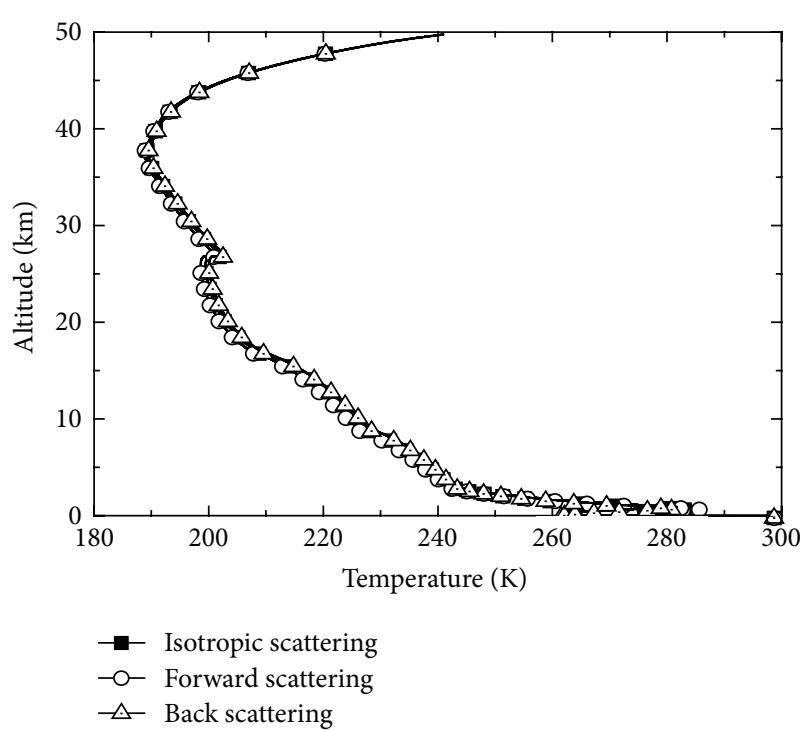

(a) Atmosphere temperature field $(0-50 \mathrm{~km})$

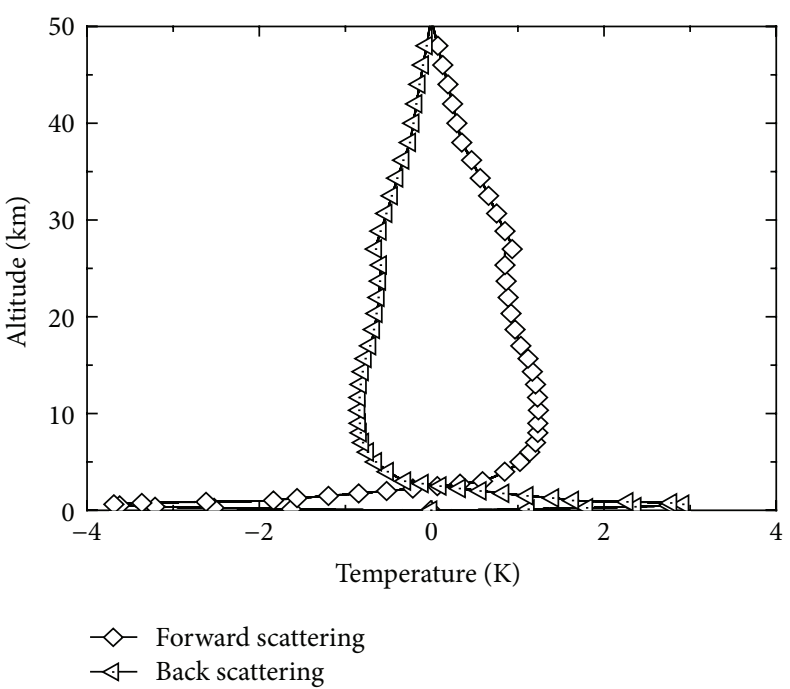

(b) Temperature difference between different scattering phase functions and isotropic scattering

FIGURE 4: Influence of scattering phase function change on atmospheric temperature.

TABLE 3: Spectral bands for the atmospheric radiative transfer model and radiative characteristic parameters under the standard dust aerosol in the height of $0-1 \mathrm{~km}$.

\begin{tabular}{lccc}
\hline Band number & $\begin{array}{c}\text { Wavelength } \\
\text { range }(\mu \mathrm{m})\end{array}$ & $\begin{array}{c}\text { Optical } \\
\text { thickness }\end{array}$ & Albedo \\
\hline 1 & $0.2 \sim 0.5$ & 92.92 & 0.86 \\
2 & $0.5 \sim 0.8$ & 12.30 & 0.88 \\
3 & $0.8 \sim 1.25$ & 4.42 & 0.86 \\
4 & $1.25 \sim 1.59$ & 43.28 & 0.80 \\
5 & $1.59 \sim 2.22$ & 4.27 & 0.48 \\
6 & $2.22 \sim 3.45$ & 23.65 & 0.07 \\
7 & $3.45 \sim 5.0$ & 93.93 & 0.02 \\
8 & $5.0 \sim 10.0$ & 353.22 & 0.00 \\
9 & $10.0 \sim 11.11$ & 12.51 & 0.11 \\
10 & $11.11 \sim 12.99$ & 1.62 & 0.45 \\
11 & $12.99 \sim 20.0$ & 35.29 & 0.01 \\
12 & $20.0 \sim 50.0$ & 951.33 & 0.00 \\
\hline
\end{tabular}

particle size distribution function is the multilognormal distribution.

From the results in the figure we can see that as the different scattering effect of the aerosol particles, their influence on the temperature is greatly different. Compared with dust aerosol, the marine and water-soluble aerosol particles have stronger scattering effect. They will scatter upwards more radiation energy and decrease the atmospheric temperature. The soot particles have stronger absorption and their existence will cause the atmospheric temperature increasing.

3.2. The Influence of the Scattering Phase Function Variation. The aerosol particles scattering characteristics are considered as forward and back scatter (the scattering phase functions are $\Phi_{1}(\Theta)$ and $\Phi_{2}(\Theta)$, resp.; see (16) and (17)). Other parameters are not changed. The results are shown in Figure 4:

$$
\begin{aligned}
& \Phi_{1}(\Theta)=1+P_{1}(\cos \Theta), \\
& \Phi_{2}(\Theta)=1-P_{1}(\cos \Theta) .
\end{aligned}
$$

It can be seen from Figure 4(a) that, compared with the anisotropic scattering, the variation in the scattering phase functions causes the atmospheric temperature to change. But the effect is not obvious. Figure 4(b) shows the difference value between the atmospheric temperature fields and the isotropic scattering with different scattering phase functions. From the figure we can see that the forward and back scatters have opposite effect on the atmospheric temperature fields but the effect degree is similar.

3.3. The Influence of the Aerosol Particles Concentration. The aerosol particles transport with the tropospheric movement will cause the aerosol particles concentrations to vary. In Figure 5 we consider the influence of the variation in total particles number concentrations, and the particles number concentrations are increased or decreased by $50 \%$. So the radiation characteristics of the aerosol particles are changed, the atmospheric energy balance is affected, and then the atmospheric temperature fields are influenced. From the results it can be seen that the variation in aerosol particles concentrations can affect the atmospheric temperature but not obviously compared with other factors.

3.4. The Influence of the Height Distribution of Aerosol Particles. We investigate the effect of the medium layer height on the aerosol particles size distribution; that is, the atmospheric radiation temperature fields change with the aerosol particles 
TABLE 4: Complex refractive index of different wavelength for different aerosol types [19].

\begin{tabular}{|c|c|c|c|c|c|c|c|c|}
\hline \multirow{2}{*}{$\lambda$} & \multicolumn{2}{|c|}{ Dust } & \multicolumn{2}{|c|}{ Water-soluble } & \multicolumn{2}{|c|}{ Marine } & \multicolumn{2}{|c|}{ Soot } \\
\hline & $n$ & $k$ & $n$ & $k$ & $n$ & $k$ & $n$ & $k$ \\
\hline 0.200 & 1.530 & 0.070 & 1.530 & 0.070 & 1.429 & $2.87 E-5$ & 1.500 & 0.350 \\
\hline 0.250 & 1.530 & 0.030 & 1.530 & 0.030 & 1.404 & $1.45 E-6$ & 1.620 & 0.450 \\
\hline 0.300 & 1.530 & 0.008 & 1.530 & 0.008 & 1.395 & $5.83 E-6$ & 1.740 & 0.470 \\
\hline 0.337 & 1.530 & 0.008 & 1.530 & 0.005 & 1.392 & $1.20 E-7$ & 1.750 & 0.470 \\
\hline 0.400 & 1.530 & 0.008 & 1.530 & 0.005 & 1.385 & $9.90 E-9$ & 1.750 & 0.460 \\
\hline 0.488 & 1.530 & 0.008 & 1.530 & 0.005 & 1.382 & $6.41 E-9$ & 1.750 & 0.450 \\
\hline 0.515 & 1.530 & 0.008 & 1.530 & 0.005 & 1.381 & $3.70 E-9$ & 1.750 & 0.450 \\
\hline 0.550 & 1.530 & 0.008 & 1.530 & 0.006 & 1.381 & $4.26 E-9$ & 1.750 & 0.440 \\
\hline 0.633 & 1.530 & 0.008 & 1.530 & 0.006 & 1.377 & $1.62 E-8$ & 1.750 & 0.430 \\
\hline 0.694 & 1.530 & 0.008 & 1.530 & 0.007 & 1.376 & $5.04 E-8$ & 1.750 & 0.430 \\
\hline 0.860 & 1.520 & 0.008 & 1.520 & 0.012 & 1.372 & $1.09 E-6$ & 1.750 & 0.430 \\
\hline 1.060 & 1.520 & 0.008 & 1.520 & 0.017 & 1.367 & $6.01 E-5$ & 1.750 & 0.440 \\
\hline 1.300 & 1.460 & 0.008 & 1.510 & 0.020 & 1.365 & $1.41 E-4$ & 1.760 & 0.450 \\
\hline 1.536 & 1.400 & 0.008 & 1.510 & 0.023 & 1.359 & $2.43 E-4$ & 1.770 & 0.460 \\
\hline 1.800 & 1.330 & 0.008 & 1.460 & 0.017 & 1.351 & $3.11 E-4$ & 1.790 & 0.480 \\
\hline 2.000 & 1.260 & 0.008 & 1.420 & 0.008 & 1.347 & 0.00107 & 1.800 & 0.490 \\
\hline 2.250 & 1.220 & 0.009 & 1.420 & 0.010 & 1.334 & $8.50 E-4$ & 1.810 & 0.500 \\
\hline 2.500 & 1.180 & 0.009 & 1.420 & 0.012 & 1.309 & 0.00239 & 1.820 & 0.510 \\
\hline 2.700 & 1.180 & 0.013 & 1.400 & 0.055 & 1.249 & 0.0156 & 1.830 & 0.520 \\
\hline 3.000 & 1.160 & 0.012 & 1.420 & 0.022 & 1.439 & 0.197 & 1.840 & 0.540 \\
\hline 3.200 & 1.220 & 0.010 & 1.430 & 0.008 & 1.481 & 0.0669 & 1.860 & 0.540 \\
\hline 3.392 & 1.260 & 0.013 & 1.430 & 0.007 & 1.439 & 0.0151 & 1.870 & 0.550 \\
\hline 3.500 & 1.280 & 0.011 & 1.450 & 0.005 & 1.423 & 0.00717 & 1.880 & 0.560 \\
\hline 3.750 & 1.270 & 0.011 & 1.452 & 0.004 & 1.398 & 0.0029 & 1.900 & 0.570 \\
\hline 4.000 & 1.260 & 0.012 & 1.455 & 0.005 & 1.388 & 0.00369 & 1.920 & 0.580 \\
\hline 4.500 & 1.260 & 0.014 & 1.460 & 0.013 & 1.377 & 0.00997 & 1.940 & 0.590 \\
\hline 5.000 & 1.250 & 0.016 & 1.450 & 0.012 & 1.366 & 0.00957 & 1.970 & 0.600 \\
\hline 5.500 & 1.220 & 0.021 & 1.440 & 0.018 & 1.333 & 0.00931 & 1.990 & 0.610 \\
\hline 6.000 & 1.150 & 0.037 & 1.410 & 0.023 & 1.306 & 0.0796 & 2.020 & 0.620 \\
\hline 6.200 & 1.140 & 0.039 & 1.430 & 0.027 & 1.431 & 0.0691 & 2.030 & 0.625 \\
\hline 6.500 & 1.130 & 0.042 & 1.460 & 0.033 & 1.374 & 0.0294 & 2.040 & 0.630 \\
\hline 7.200 & 1.400 & 0.055 & 1.400 & 0.070 & 1.343 & 0.0249 & 2.060 & 0.650 \\
\hline 7.900 & 1.150 & 0.040 & 1.200 & 0.065 & 1.324 & 0.0279 & 2.120 & 0.670 \\
\hline 8.200 & 1.130 & 0.074 & 1.010 & 0.100 & 1.324 & 0.0308 & 2.130 & 0.680 \\
\hline 8.500 & 1.300 & 0.090 & 1.300 & 0.215 & 1.336 & 0.0336 & 2.150 & 0.690 \\
\hline 8.700 & 1.400 & 0.100 & 2.400 & 0.290 & 1.366 & 0.0356 & 2.160 & 0.690 \\
\hline 9.000 & 1.700 & 0.140 & 2.560 & 0.370 & 1.373 & 0.0365 & 2.170 & 0.700 \\
\hline 9.200 & 1.720 & 0.150 & 2.200 & 0.420 & 1.356 & 0.0371 & 2.180 & 0.700 \\
\hline 9.500 & 1.730 & 0.162 & 1.950 & 0.160 & 1.339 & 0.0368 & 2.190 & 0.710 \\
\hline 9.800 & 1.740 & 0.162 & 1.870 & 0.095 & 1.324 & 0.0388 & 2.200 & 0.715 \\
\hline 10.000 & 1.750 & 0.162 & 1.820 & 0.090 & 1.310 & 0.0406 & 2.210 & 0.720 \\
\hline 10.591 & 1.620 & 0.120 & 1.760 & 0.070 & 1.271 & 0.0522 & 2.220 & 0.730 \\
\hline 11.000 & 1.620 & 0.105 & 1.720 & 0.050 & 1.246 & 0.0731 & 2.230 & 0.730 \\
\hline 11.500 & 1.590 & 0.100 & 1.670 & 0.047 & 1.227 & 0.105 & 2.240 & 0.740 \\
\hline 12.500 & 1.510 & 0.090 & 1.620 & 0.053 & 1.208 & 0.190 & 2.270 & 0.750 \\
\hline 13.000 & 1.470 & 0.100 & 1.620 & 0.055 & 1.221 & 0.223 & 2.280 & 0.760 \\
\hline 14.000 & 1.520 & 0.085 & 1.560 & 0.073 & 1.267 & 0.271 & 2.310 & 0.775 \\
\hline 14.800 & 1.570 & 0.100 & 1.440 & 0.100 & 1.307 & 0.292 & 2.330 & 0.790 \\
\hline
\end{tabular}


TABle 4: Continued.

\begin{tabular}{|c|c|c|c|c|c|c|c|c|}
\hline \multirow{2}{*}{$\lambda$} & \multicolumn{2}{|c|}{ Dust } & \multicolumn{2}{|c|}{ Water-soluble } & \multicolumn{2}{|c|}{ Marine } & \multicolumn{2}{|c|}{ Soot } \\
\hline & $n$ & $k$ & $n$ & $k$ & $n$ & $k$ & $n$ & $k$ \\
\hline 15.000 & 1.570 & 0.100 & 1.420 & 0.200 & 1.321 & 0.297 & 2.330 & 0.790 \\
\hline 16.400 & 1.600 & 0.100 & 1.750 & 0.160 & 1.407 & 0.331 & 2.360 & 0.810 \\
\hline 17.200 & 1.630 & 0.100 & 2.080 & 0.240 & 1.487 & 0.341 & 2.380 & 0.820 \\
\hline 18.000 & 1.640 & 0.115 & 1.980 & 0.180 & 1.525 & 0.341 & 2.400 & 0.825 \\
\hline 18.500 & 1.640 & 0.120 & 1.850 & 0.170 & 1.536 & 0.339 & 2.410 & 0.830 \\
\hline 20.000 & 1.680 & 0.220 & 2.120 & 0.220 & 1.560 & 0.324 & 2.450 & 0.850 \\
\hline 21.300 & 1.770 & 0.280 & 2.060 & 0.230 & 1.568 & 0.318 & 2.460 & 0.860 \\
\hline 22.500 & 1.900 & 0.280 & 2.000 & 0.240 & 1.579 & 0.316 & 2.480 & 0.870 \\
\hline 25.000 & 1.970 & 0.240 & 1.880 & 0.280 & 1.596 & 0.313 & 2.510 & 0.890 \\
\hline 27.900 & 1.890 & 0.320 & 1.840 & 0.290 & 1.612 & 0.320 & 2.540 & 0.910 \\
\hline 30.000 & 1.800 & 0.420 & 1.820 & 0.300 & 1.614 & 0.320 & 2.570 & 0.930 \\
\hline 35.000 & 1.900 & 0.500 & 1.920 & 0.400 & 1.597 & 0.383 & 2.630 & 0.970 \\
\hline 40.000 & 2.100 & 0.600 & 1.860 & 0.500 & 1.582 & 0.561 & 2.690 & 1.000 \\
\hline
\end{tabular}

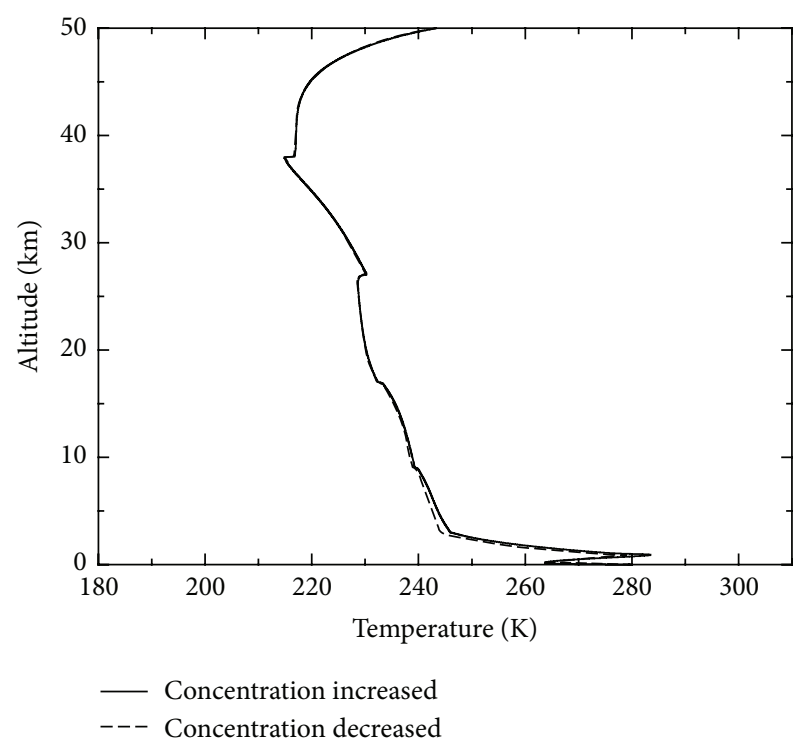

(a) Atmosphere temperature field $(0-50 \mathrm{~km})$

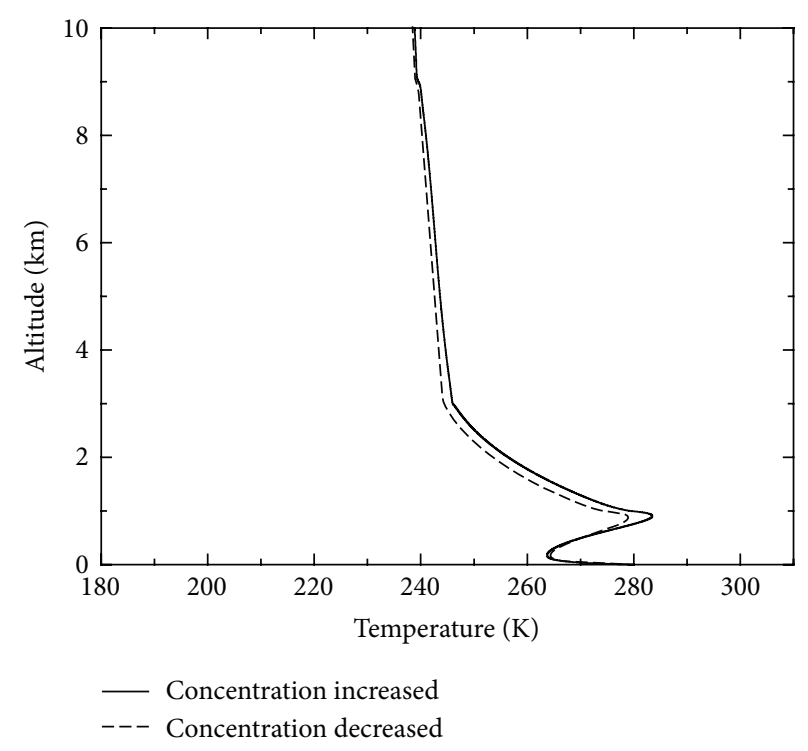

(b) Atmosphere temperature field $(0-10 \mathrm{~km})$

FIGURE 5: Influence of the aerosol concentration on atmospheric temperature.

being in different height medium layers. The calculation conditions are as follows: the aerosol particles distribute in the height of $0 \sim 1 \mathrm{~km}, 1 \sim 3 \mathrm{~km}$, and $3 \sim 9 \mathrm{~km}$, respectively. The calculation results are in Figure 6.

From the calculation results in Figure 6 it can be seen that, with the aerosol particles transporting to the high altitude, the atmospheric temperature is affected greatly if the radiation characteristics of the aerosol particles have no changes. With the distribution height of the aerosol particles increasing, the medium temperature above the medium layer in which the aerosol particles exist increases greatly but the below temperature decreases obviously. This phenomenon shows the scattering effect of the aerosol particles on the solar radiation energy, that is, as the scattering effect of the atmospheric aerosols, most of the solar radiation energy to the ground is scattered back to the high altitude and cannot reach lower atmosphere, which causes the lower atmospheric temperature to decrease to about $200 \mathrm{~K}$.

\section{Conclusion}

We analyzed the influence of the aerosol particles characteristics on the atmospheric radiation transfer model. The results showed the different effect of aerosol types on the atmospheric radiation. The dust, marine, and water-soluble 


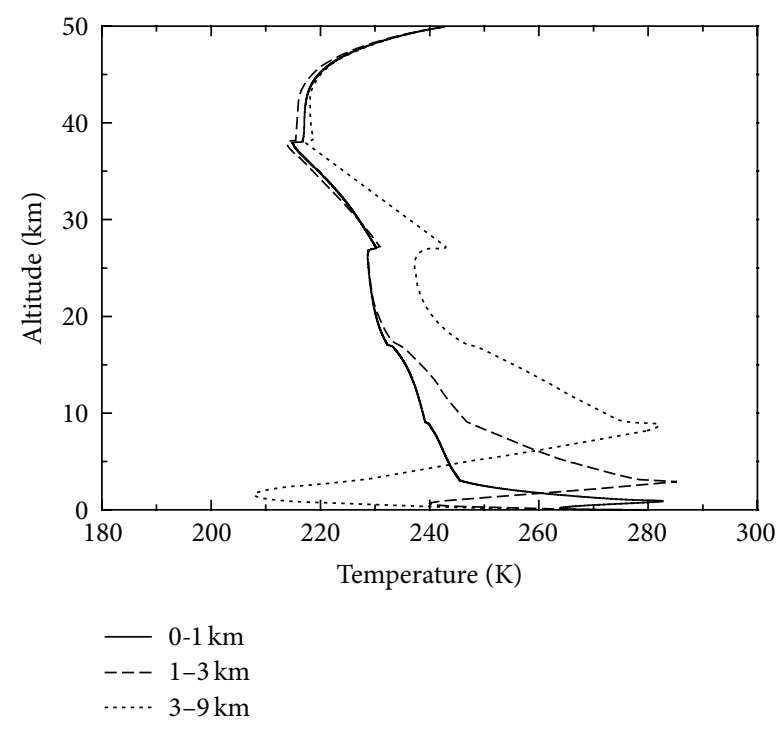

Figure 6: Influence of temperature fields on different aerosol distribution altitude.

aerosols had negative effects on the atmospheric absorption, but the soot aerosol could increase the atmospheric absorption. The scattering phase functions affected the ground temperature. The variation of the aerosol particles distribution height could determine the atmospheric temperature; that is, the ground temperature decreased greatly with the aerosol particles existing in the high altitude stable.

\section{Conflict of Interests}

The authors declare that there is no conflict of interests regarding the publication of this paper.

\section{Acknowledgments}

This work was supported by the Foundation for Innovative Research Groups of the National Natural Science Foundation of China (Grant no. 51121004) and the National Natural Science Foundation of China (Grant nos. 51176040 and 51276193). A very special acknowledgement is made to the editors and referees who made important comments to improve this paper.

\section{References}

[1] M. Schulz, C. Textor, S. Kinne et al., "Radiative forcing by aerosols as derived from the AeroCom present-day and preindustrial simulations," Atmospheric Chemistry and Physics, vol. 6, pp. 5225-5246, 2006.

[2] U. Lohmann and J. Feichter, "Global indirect aerosol effects: a review," Atmospheric Chemistry and Physics, vol. 5, pp. 715-737, 2005.

[3] I. Koren, Y. J. Kaufman, L. A. Remer, and J. V. Martins, "Measurement of the effect of Amazon smoke on inhibition of cloud formation," Science, vol. 303, no. 5662, pp. 1342-1345, 2004.
[4] W. W. Qing, R. S. Chen, and W. M. Sun, "Estimation of global radiation in China and comparison with satellite product," Environmental Earth Sciences, vol. 70, no. 4, pp. 1681-1687, 2013.

[5] X. S. Wang, "Metal geochemical and mineral magnetic characterization of the $<2.5 \mathrm{~lm}$ fraction of urban soils in Xuzhou (China)," Environmental Earth Sciences, vol. 71, no. 8, pp. 34913501, 2014.

[6] L. David, B. Nacer, B. Pascal, and J. Gérard, "Transient radiative and conductive heat transfer in non-gray semitransparent twodimensional media with mixed boundary conditions," Heat and Mass Transfer, vol. 42, no. 4, pp. 322-337, 2006.

[7] S. Dembele, K. L. M. Lima, and J. X. Wen, "Assessment of an unstructured exponential scheme discrete ordinates radiation model for non-gray media," Heat and Mass Transfer, vol. 47, no. 11, pp. 1349-1362, 2011.

[8] P. Talukdar, F. V. Issendorff, D. Trimis, and C. J. Simonson, "Conduction-radiation interaction in 3D irregular enclosures using the finite volume method," Heat and Mass Transfer, vol. 44, no. 6, pp. 695-704, 2008.

[9] C.-Y. Wu, "Monte Carlo simulation of radiative transfer in a refractive layered medium," Heat and Mass Transfer, vol. 46, no. 6, pp. 607-613, 2010.

[10] J. H. Jeans, "The equations of radiative transfer of energy," Monthly Notices Royal Astronomical Society, vol. 78, pp. 28-36, 1917.

[11] B. Davison, Neutron Transport Theory, Oxford University Press, London, UK, 1958.

[12] V. Kourganoff, Basic Methods in Transfer Problems, Dover, New York, NY, USA, 1963.

[13] H. Khouaja, D. R. Edwards, and N. Tsoulfanidis, "Spherical harmonics-finite element treatment of neutron transport in cylindrical geometry," Annals of Nuclear Energy, vol. 24, no. 7, pp. 515-531, 1997.

[14] E. W. Larsen, G. Thömmes, A. Klar, M. Seaïd, and T. Götz, "Simplified $P_{n}$ approximations to the equations of radiative heat transfer and application," Journal of Computational Physics, vol. 183, no. 2, pp. 652-675, 2002.

[15] R. G. McClarren and C. D. Hauck, "Robust and accurate filtered spherical harmonics expansions for radiative transfer," Journal of Computational Physics, vol. 229, no. 16, pp. 5597-5614, 2010.

[16] M. F. Modest, Radiative Heat Transfer, Academic Press, Amsterdam, The Netherlands, 2nd edition, 2003.

[17] R. Siegel and C. M. Spuckler, "Refractive index effects on radiation in an absorbing, emitting, and scattering laminated layer," Journal of Heat Transfer, vol. 115, pp. 194-200, 1993.

[18] Q. Z. Yu, Principles of Radiative Heat Transfer, Harbin Institute of Technology Press, Harbin, China, 2000.

[19] G. Y. Shi, Atmospheric Radiology, Science Press, Beijing, China, 2007. 


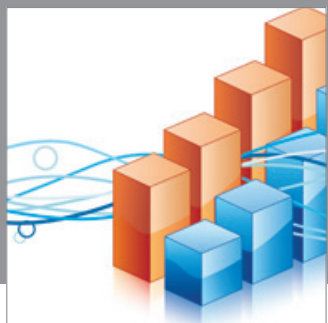

Advances in

Operations Research

mansans

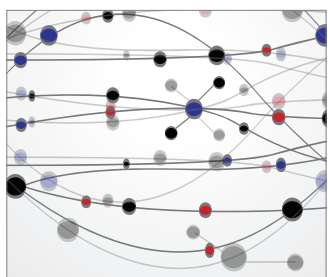

The Scientific World Journal
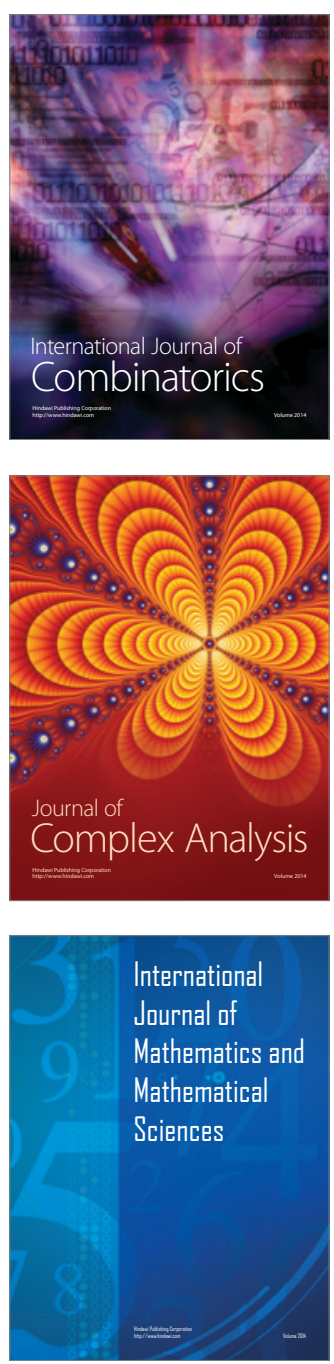
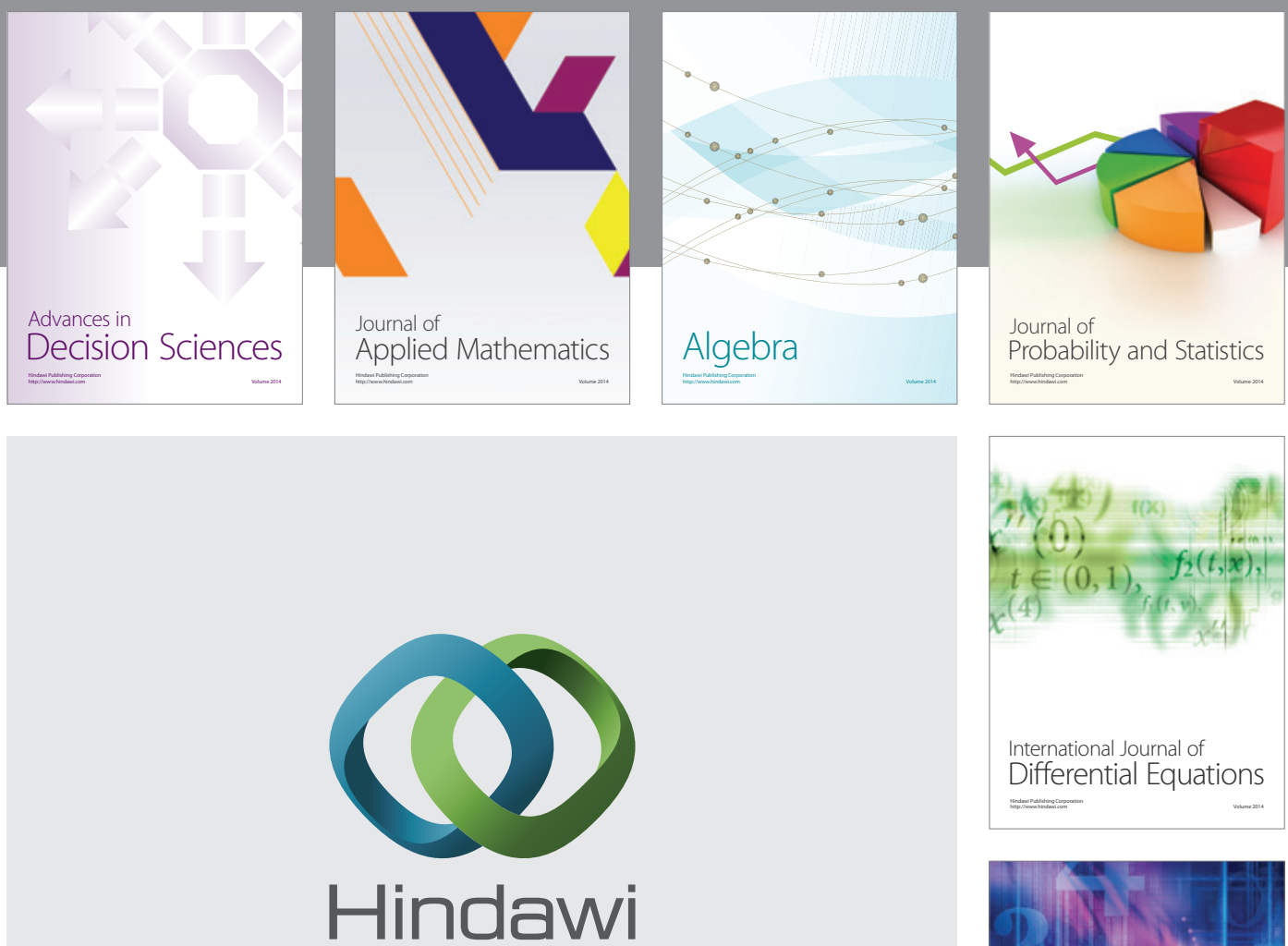

Submit your manuscripts at http://www.hindawi.com
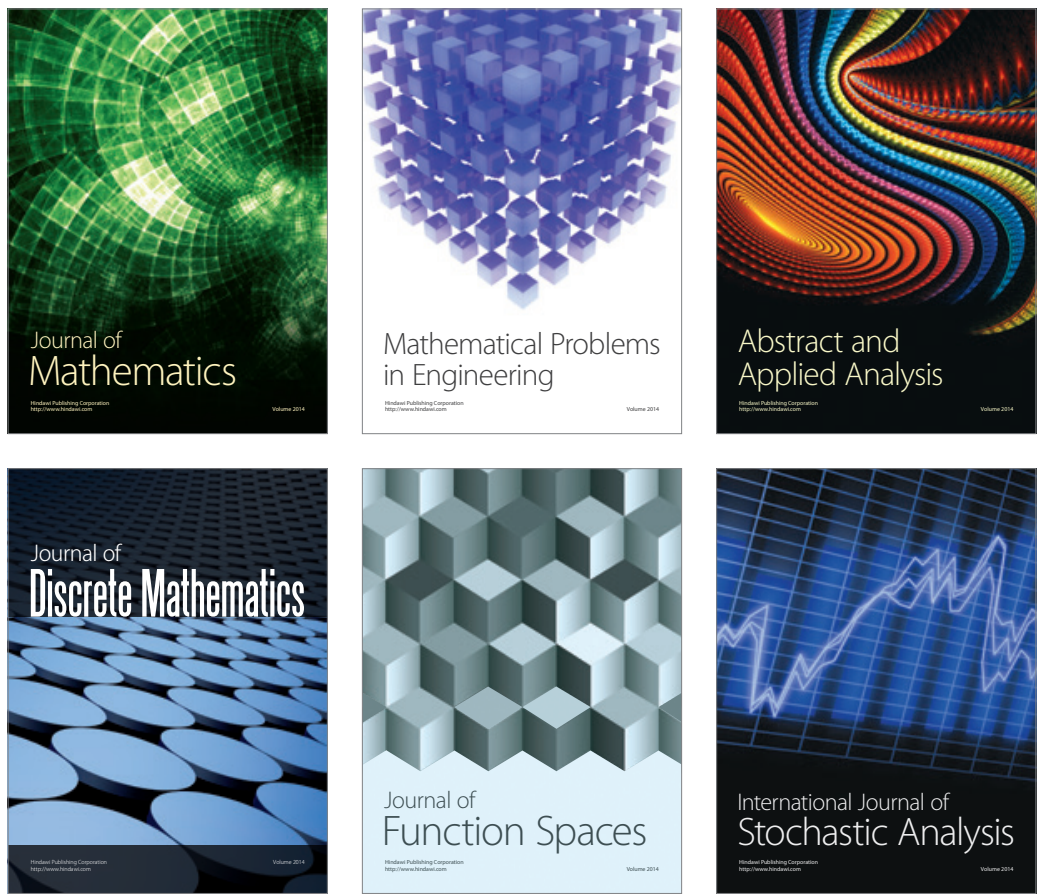

Journal of

Function Spaces

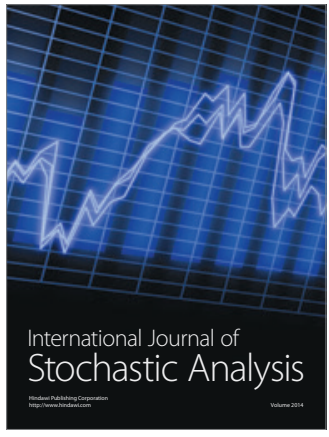

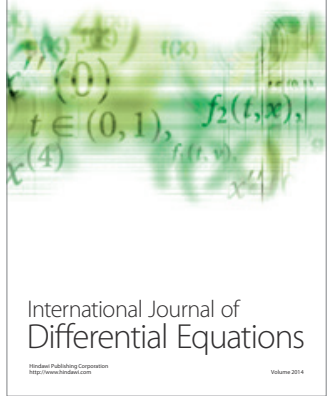
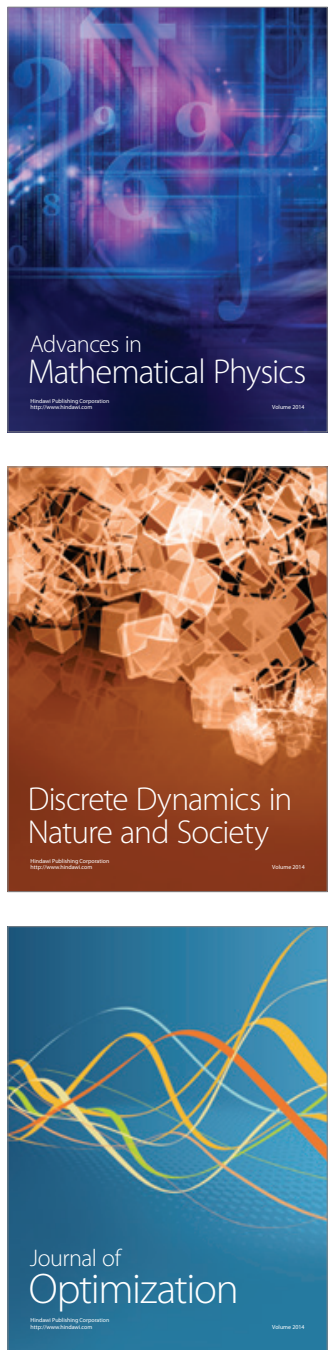